\title{
Journal of Neurology: ready for continued success
}

\author{
Roger A. Barker · Massimo Filippi · \\ Michael Strupp
}

Published online: 18 December 2012

(c) Springer-Verlag Berlin Heidelberg 2012

We thought it might be useful to update our readers, authors and reviewers on some new developments in the Journal of Neurology (JoN). As many of you know the JoN has a long history and in recent times it has been linked to the European Neurological Society (ENS). Efforts continue to focus on not only further development of the JoN but also our position with respect to other neurological journals. As such, we thought this might be a useful time to rethink some aspects of the JoN to try and improve on its already impressive achievements to date. This is becoming more of an issue as the popularity of the journal increases with more than 1,500 submissions in 2012 compared to 917 in 2009. This has also brought about a higher rejection rate for manuscripts, $82 \%$ in 2012 compared to $66 \%$ in 2009, but also has necessitated a faster editorial turnaround time for making a final decision on a paper which has dropped from 60 days in 2009 to 23 days in 2012.

In order to try and improve on this, we have decided to provide some further guidelines to help authors and reviewers. For those wishing to submit papers, we have

R. A. Barker $(\bowtie)$

Cambridge Centre for Brain Repair, University of Cambridge,

The E.D. Adrian Building, Forvie Site, Robinson Way,

Cambridge CB2 2PY, UK

e-mail: rab46@cam.ac.uk

\section{Filippi}

Neuroimaging Research Unit, Institute of Experimental Neurology, Division of Neuroscience, San Raffaele Scientific Institute and Vita-Salute San Raffaele University, Via Olgettina 60, 20132 Milan, Italy

e-mail: filippi.massimo@hsr.it

\section{Strupp}

Department of Neurology, University Hospital Munich, Campus Grosshadern, Marchioninistrasse 15, 81377 Munich, Germany e-mail: Michael.Strupp@med.uni-muenchen.de decided that the article should be relevant to clinical neurology and, as such, manuscripts on experimental studies in animal models of disease will not be considered by JoN. In addition, the clinical studies should in some way move the field forward, be clinically relevant and well designed, i.e., preferably prospective randomized controlled trials; underpowered, anecdotal studies will have less chance of being published. Further, Review Articles on clinical relevant topics are very welcome and highly appreciated by the readership.

We are also keen to get papers through the review process as fast as possible and publish them in a timely and attractive fashion. We are therefore asking referees to review the paper within 14 days (not 21 as it is at present) and in cases where the paper needs to be revised, whether it also needs to be re-reviewed. We are also asking authors to ensure that their paper is no longer than 4,000 words with a maximum of five figures or tables and 40 references. We are also hoping to make the titles of the papers shorter and more precise, and restructure the contents page to make it more obvious what category of sub-speciality each paper falls within in every issue of the Journal.

In terms of new additions to the JoN, we have decided to have a new section called "Neurological Progress" and this in part will replace the "Medical Progress Section" that we have been featuring for the last two years. This will contain short reviews on topics that have either been the subject of several papers within the JoN in recent times or been the subject of a recent workshop or meeting. These articles will be invited and we are hopeful that they will be published very quickly so that our readers will be informed of new developments and ideas in the world of neurology. The section "Techniques in Clinical Science" will be enhanced; the new editor responsible for this section will be Massimo Filippi. 
Finally, we would like to thank you for your continued support and help with the JoN and especially all our reviewers who put in so much time and effort to make the journal what it is. Those who have done this can be found on our website http://www.jon.springer.de, and those asterisked have been our star performers, so a very special thanks to them. 